\title{
Assessing Risks of Impaired Healing and Osteomyelitis Associated with Bisphosphonates Using FDA Adverse Event Reporting System (FAERS)
}

Xiaodong Feng ${ }^{1 *}$, Amie Cai ${ }^{2}$, Ana Hincapie ${ }^{1}$, Kevin Dong ${ }^{1}$, Wendy Chaing ${ }^{1}$, Tibebe Woldemariam ${ }^{1}, \mathrm{Kevin} \mathrm{Yamashiro}^{3}$ and Bin Guan ${ }^{4}$

${ }^{1}$ California Northstate University College of Pharmacy, Rancho Cordova, CA, USA

${ }^{2}$ Department of Molecular and Cell Biology, University of Berkeley, Berkeley, CA, USA

${ }^{3}$ University of Southern California School of Pharmacy, Los Angeles, CA, USA

${ }^{4}$ Kaiser South Sacramento, Sacramento, CA, USA

\begin{abstract}
Objective: Bisphosphonates are the leading class of drugs to prevent and treat osteoporosis. Numerous cases of bisphosphonates related to bone necrosis and bone fractures have been reported. In this study the risks of impaired healing and osteomyelitis associated with bisphosphonates were assessed using case reports submitted to the US Food and Drug Administration (FDA) Adverse Event Reporting System (FAERS).
\end{abstract}

Methods: Using FAERS the adverse events reports from the first quarter of 2004 to the second quarter of 2012 associated with bisphosphonates and non-bisphosphonates anti-osteoporosis drugs were generated and evaluated. Standardized pharmacovigilance tools were applied to detect the signal of impaired healing and osteoporosis.

Results: Among 14493 total reported events of impaired healing in FAERS, 49\% of the cases were associated with use of bisphophonates. Substantial and pharmacovigilantly significant signals of impaired healing ( $P R R=13.39)$ and osteomyelitis $(P R R=7.06)$ were detected. There was less risk of impaired healing $(P R R=4.8)$ and osteomyelitis $(P R R=1.61)$ associated with ibandronate $(P R R=4.80)$ compared to other bisphosphonates. Interestingly weak but significant signals of impaired healing $(P R R=3.40)$ and osteomyelitis $(P R R=2.38)$ were also detected for denosumab. There were no significant risks of impaired healing $(P R R=1.85)$ and osteomyelitis $(P R R=0.25)$ associated with teriparatide.

Conclusion: This study for the first time signals an increasing risk of impaired healing and osteomyelitis associated with bisphosphonates using FAERS. It has significant implication for patient safety in pharmacy practice. Considering the limitation of FAERS, such as under reporting, reporting bias and Weber-effect, this study provides goal for future large pharmacoepidemiologic studies.

Keywords: Bisphosphonates; Impaired healing; Osteomyelitis; FDA adverse event reporting system

\section{Introduction}

The Food and Drug Administration Adverse Event Reporting System (FAERS) represents one of the most successful public health information databases sponsored by the United States government to assure the safety, efficacy, and security of post market medications. The FAERS is a public media platform for consumers and clinicians to address their concerns of drug safety by reporting the adverse drug events to the Food and Drug Administration (FDA). The FAERS creates new information ecology by providing an open communication channel between consumers, clinicians and the FDA. It also has the potential to serve as a pharmacovigilance tool to identify the important issues of drug safety [1-4].

Along with environmental and genetic risk factors, drugs also play an important role in delayed wound healing. Every year thousands of drug related impaired healing events are communicated by health care consumers to their health care providers, and most of these adverse events are reported to the FDA through FAERS. Identifying risk of impaired healing associated with specific drugs is essential for wound care and also offers an essential solution for control and prevention of delayed wound healing. Van Beusekom and colleagues reported delayed healing associated with paclitaxel and sirolimus eluting stents [5]. Colombres et al. recently reported that delayed epithelial healing after intravitreal bevacizumab, a monoclonal blocking antibody for vascular endothelial growth factor (VEGF). The FDA recommends discontinuing bevacizumab in patients with wound dehiscence, and discontinuing bevacizumab at least 28 days prior to elective surgery [6].
In addition, FDA recommends not initiating bevacizumab for at least 28 days after surgery and until the surgical wound is fully healed [6]. However, the risks of impaired healing associated with bisphosphonates are controversial and poorly defined.

Bisphosphonates are synthetic potent inhibitors of osteoclastmediated bone resorption, and they are mainly used for the treatment and prevention of osteoporosis in both men and women. Alendronate was the first bisphosphonate approved by the Food and Drug Administration (FDA) in 1995 for the treatment and prevention of osteoporosis, and indicated for the treatment of symptomatic Paget's disease of bone. Risedronate, pamidronate, zoledronic acid, ibandronate, tiludronate and etidronate are other bisphosphonates currently approved in the US market. Etidronate and tiludronate are non-nitrogen containing bisphosphonates indicated for the treatment of symptomatic Paget's disease of bone. Etidronate was also approved for the prevention and treatment of heterotopic ossification following

*Corresponding author: Xiaodong Feng, California Northstate University College of Pharmacy, 10811 International Dr., Rancho Cordova, CA-9570, USA, Tel: (916) 5031812; Fax: (916) 631-8127; E-mail: xfeng@cnsu.edu

Received January 23, 2015; Accepted February 17, 2015; Published February 23, 2015

Citation: Feng X, Cai A, Hincapie A, Dong K, Chaing W, et al. (2015) Assessing Risks of Impaired Healing and Osteomyelitis Associated with Bisphosphonates Using FDA Adverse Event Reporting System (FAERS). J Pharmacovigilance 3: 158. doi:10.4172/2329-6887.1000158

Copyright: (c) 2015 Feng X, et al. This is an open-access article distributed under the terms of the Creative Commons Attribution License, which permits unrestricted use, distribution, and reproduction in any medium, provided the original author and source are credited. 
Citation: Feng X, Cai A, Hincapie A, Dong K, Chaing W, et al, et al. (2015) Assessing Risks of Impaired Healing and Osteomyelitis Associated with Bisphosphonates Using FDA Adverse Event Reporting System (FAERS). J Pharmacovigilance 3: 158. doi:10.4172/2329-6887.1000158

Page 2 of 6

total hip replacement or due to spinal cord injury. Intravenous zoledronic acid and pamidronate are also used to manage bone symptoms for metastatic cancer patients [7].

Bisphosphonates are relative safe and effective, however recent uncommon adverse effects associated with the use of bisphosphonates have been emerging. Atrial fibrillation, risk of esophageal cancer, and development of atypical fractures are some other potential serious post-marketing new adverse effects reported to be associated with pharmacotherapy of biosphosphonates $[8,9]$. The risks of impaired healing and osteomyelitis associated with bisphosphonates pharmacotherapy are currently under debate. One of the major challenges in orthopedics is the osteoporotic fracture management. Stein and colleagues demonstrate in a meta-analysis that bisphosphonates and vitamin $D$ treatment during the first year after solid organ transplant reduce the risk of fracture and vertebral fractures [10]. In contrast, using FAERS database Edwards and colleagues detected significant risks of nonhealing femoral fractures associated with bisphosphonates [11]. The present study was performed to assess the risks of impaired healing and osteomyelitis associated with bisphosphonates and nonbisphosphonates anti-osteoporosis drugs based on millions of case reports of adverse events submitted to FAERS.

\section{Methods}

\section{Data resources}

Using the public version of FDA Adverse Events Reporting System (FAERS) database, all the adverse events reported to FDA $(15,515,692$ cases) from the first quarter of 2004 to the second quarter of 2012 were obtained and evaluated. Data were obtained from the FAERS which contains spontaneous adverse event case reports filed from 2004 to 2012 by the consumers, health care providers and pharmaceutical manufacturers regarding identifiable, but anonymous, patients. The new cases of suspected adverse events are released to the public every quarter and the reports include seven data sets: patient demographic and administrative information; drug information; adverse events; patient outcomes; report sources; drug therapy start and end dates; and indications for use.

\section{Drugs selected for analysis}

All the FDA approved bisphosphonates on the USA market were selected for this study. The drugs selected were alendronate, risedronate, pamidronate, ibandronate, zoledronic acid, tiludronate and etidronate. When the database reports were generated, the drug name variant for each drug, such as brand names, generic name, chemical names, misspelling names and names with dosage descriptions, were used to consolidate the reports into one form under generic name. For control purpose, some other drugs indicated for bone disorders were also selected for this study. The controls of non-bisphosphonate antiosteoporosis drugs were denosumab and teriparatide.

\section{Data processing}

Relational databases were first created from the ASCII files in the FAERS using Microsoft Office Access and Oracle. Then the data were sorted and analyzed with Microsoft Excel and Oracle analytic tools. The predefined events of interest were impaired healing, and osteomyelitis. Using the FAERS public database, the ADRs associated with bisphosphonates and control drugs were generated and evaluated. The adverse events involving combination drugs were not combined with monotherapy adverse events.

\section{Statistical analysis}

Spontaneous adverse events reports associated with bisphosphonates treatment were acquired from the FAERS database using standard pharmacovigilance algorithms. To detect risk signals with alarming reporting frequencies, the proportional reporting ratio (PRR) and the reporting odds ratio (ROR) were calculated on the basis of all adverse events associated with all drugs reported in the FAERS from the first quarter of 2004 to the second quarter of 2012. Two by two contingency tables for impaired healing events associated with or without the presence of bisphosphonates were created to calculate PRR and ROR including a two sided $95 \%$ confidence interval (95\% CI). A signal for alarm was considered for a PRR $>2$ or ROR $>2$, and if the lower bound of $95 \%$ CI for ROR was greater than 1 [12-16]. Since age and sex are associated with osteomyelitis and impaired healing and are unevenly distributed among exposed and unexposed subjects, confounding and effect modification were tested. Effect modification was tested by the Breslow-Day test for homogeneity between odds ratios after stratified analyses. Confounding was evaluated by MantelHaenszel common odds ratios analyses.

\section{Results}

\section{Increasing trend of impaired healing cases reported in the FAERS}

From 2004 to 2011, the trend of impaired healing adverse events associated with pharmacotherapy has been steadily climbing (Figure 1). In 2004, only 572 cases of impaired healing were reported to FAERS, and they only counted as $0.0519 \%$ of the total reported adverse events cases. Surprisingly after seven years, the total cases increased more than 5 times to 2960 cases, in 2011, and the ratio of impaired healing cases over total adverse events was almost doubled compared to 2004. Table 1 calculated the total cases of impaired healing reported to the FAERS from the first quarter of 2004 to the second quarter of 2012 with details for each quarter (Table 1). It strongly suggested that the cases of impaired healing associated with drug treatments have been steadily increasing over the previous decade.

\section{Signals of impaired healing associated with bisphosphonates}

The impaired healing adverse events were commonly reported by patients who were treated by bisphosphonates, such as alendronate, pamidronate, risedronate, zoledronic acid, ibandronate, tiludronate

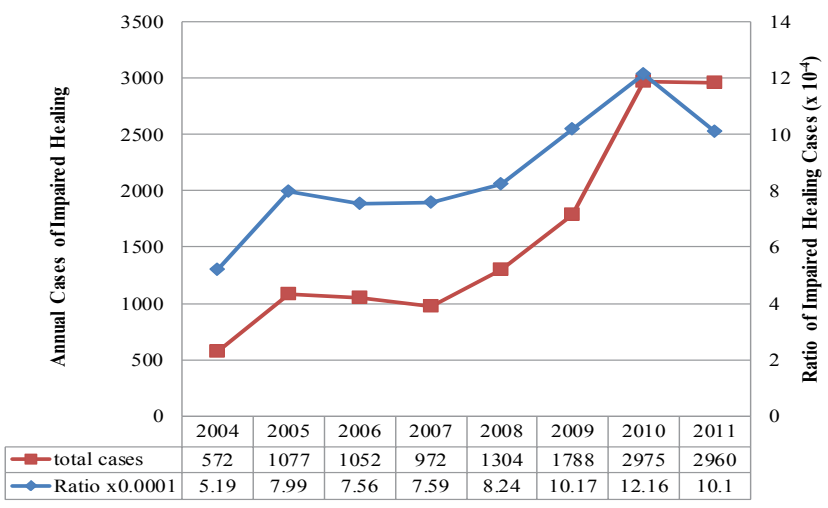

Figure 1: Increasing trend of impaired healing reported to the FAERS from 2004 to 2011. The data were obtained from the FAERS from 2004 to 2011. The total annual cases of impaired healing reported to the FAERS and the ratio of impaired healing cases over total adverse events reported to the AERS were calculated. 
Citation: Feng X, Cai A, Hincapie A, Dong K, Chaing W, et al, et al. (2015) Assessing Risks of Impaired Healing and Osteomyelitis Associated with Bisphosphonates Using FDA Adverse Event Reporting System (FAERS). J Pharmacovigilance 3: 158. doi:10.4172/2329-6887.1000158

Page 3 of 6

\begin{tabular}{|c|c|c|c|c|}
\hline \multirow[b]{2}{*}{ Year } & \multicolumn{4}{|c|}{$\begin{array}{l}\text { Total Adverse Drug Events } \\
\text { (Impaired Healing ) }\end{array}$} \\
\hline & Quarter 1 & Quarter 2 & Quarter 3 & Quarter 4 \\
\hline 2004 & 264,771 (127) & $254,444(162)$ & $291,222(133)$ & 292,139 (150) \\
\hline 2005 & $313,984(152)$ & $337,454(365)$ & $336,320(289)$ & $360,036(271)$ \\
\hline 2006 & $409,360(248)$ & 389,707 (290) & $286,316(250)$ & $306,109(264)$ \\
\hline 2007 & $301,385(214)$ & $298,589(207)$ & $315,283(238)$ & 365,659 (313) \\
\hline 2008 & $366,706(329)$ & $404,433(269)$ & $402,637(324)$ & $408,726(382)$ \\
\hline 2009 & $379,450(321)$ & $414,777(398)$ & $454,509(436)$ & $510,140(633)$ \\
\hline 2010 & $507,210(661)$ & $550,274(710)$ & $736,612(751)$ & $653,301(853)$ \\
\hline 2011 & $661,345(691)$ & $751,821(747)$ & $742,706(665)$ & $774,170(857)$ \\
\hline 2012 & $838,707(925)$ & $83,5390(868)$ & No data & No data \\
\hline
\end{tabular}

Table 1: Impaired Healing Cases and Total Adverse Events Cases Reported in FAERS (First 2004-Quarter to Second 2012-Quarter).

and etidronate. The algorithm of pharmacovigilance was used for data mining from the FAERS to assess the signals of impaired healing adverse events associated with bisphosphonates. Alendronate was the first bisphosphonate approved by the FDA. From the first quarter of 2004 to the second quarter of 2012, there were 242,019 total cases of adverse events reported to the FAERS, and 2,316 of these reported cases $(0.96 \%)$ were adverse events of impaired healing. Impaired healing is one of the top 10 major adverse events reported to the FAERS along with femur fracture, osteonecrosis, fall, low turnover osteopathy, osteomyelitis, anxiety, arthralgia, depression and tooth disorder. The signal of impaired healing associated with alendronate was substantial and statistically significant $(\mathrm{PRR}=12.00, \mathrm{ROR}=12.109$; 95\% CI: 11.581-12.662) (Table 2). The control was all impaired healing cases associated with all drugs in the FERS excluding alendronate. There was no significant change for risk of impaired healing associated with alendronate plus vitamin $\mathrm{D}$ combination compared to that of alendronate monotherapy (ROR 12.109 vs. 12.157) (Table 2). Similar substantial and statistically significant pharmacovigilance signal was detected for zolendronate ( $\mathrm{PRR}=8.79, \mathrm{ROR}=8.849$, 95\% CI: 8.4799.235), pamidronate ( $\mathrm{PRR}=7.02, \mathrm{ROR}=7.057,95 \% \mathrm{CI}: 6.608-7.536)$. Interestingly, ibandronate has the lowest signal of impaired healing $(\mathrm{PRR}=4.80, \mathrm{ROR}=4.816,95 \% \mathrm{CI}: 4.207-5.514)$. The signal of impaired healing associated with eidronate was also substantial and statistically significant ( $\mathrm{PRR}=10.09$, and $\mathrm{ROR}=10.173$, 95\%CI: 5.456-18.967) (Table 2). No data was available for tiludronate. All the adverse events associated with each bisphosphonate, such as alendronate, risedronate, ibandronate, zoledronic acid, tiludronate and etidronate, were combined to assess the risks of impaired healing associated with the drug class of bisphosphonates. Figure 2 compared the signals of impaired healing associated with bisphosphonates and other antiosteoporosis drugs, such as denosumab and teriparatide. Less signal of impaired healing was detected for denosumab $(P R R=3.40, R O R=3.405$; 95\% CI: 2.618-4.429), compared to that of bisphosphonates. In contrast, teriparatide the signal of impaired healing associated with teriparatide was not substantial, and the signal was not pharmacovigilantly significant $(\mathrm{PRR}=1.85, \mathrm{ROR}=1.85,95 \% \mathrm{CI}: 1.677-2.041)$.

\section{Signals of osteomyelitis associated with bisphosphonates and other anti-osteoporosis drugs}

The signal of osteomyelitis associated with alendronate was substantial and statistically significant $(P R R=5.57, R O R=5.615 ; 95 \%$ CI: 11.635-12.678). The control was all osteomyelitis cases associated with all drugs in the FERS excluding alendronate. Similar substantial and statistically significant pharmacovigilance signal of osteomyelitis was detected for zolendronate ( $\mathrm{PRR}=6.21, \mathrm{ROR}=6.303$; 95\%CI: 8.4769.233), pamidronate $(\mathrm{PRR}=6.012, \mathrm{ROR}=6.066 ; 95 \% \mathrm{CI}$ : 5.768-6.379, $)$.
Interestingly, ibandronate has the lowest signal of impaired healing ( $\mathrm{PRR}=1.61, \mathrm{ROR}=1.61 ; 95 \% \mathrm{CI}: 1.363-1.896)$. The signal of osteomyelitis associated with etidronate was also substantial and statistically significant $(\mathrm{PRR}=2.57, \mathrm{ROR}=2.565 ; 95 \% \mathrm{CI}: 1.065-6.176)$. No data was available for tiludronate. Figure 3 compared the signals of osteomyelitis associated with bisphosphonates and other anti-osteoporosis drugs, such as denosumab and teriparatide. Alendronate plus vitamin D did not reduce the signal of osteomyelitis associated with alendronate $(\mathrm{P}=0.287)$. Weak, but significant signal of osteomyelitis was detected for denosumab ( $P R R=2.38, \mathrm{ROR}=2.372$; 95\%CI: 2.372-2.968,), compared to that of bisphosphonates $(\mathrm{PRR}=7.06, \mathrm{ROR}=7.118$; 95\% CI: 6.938 7.303).The signal of osteomyelitis associated with teriparatide was not substantial, and the signal was not pharmacovigilantly significant $(\mathrm{PRR}=0.25, \mathrm{ROR}=0.25$; 95\%CI: $0.205-0.298)$.

\section{Stratifying analysis for risk of impairing healing and osteomyelitis by age, sex and time}

Bisphosphonates are commonly prescribed for female patients with postmenopausal osteoporosis and patients over 60 year-old generally have higher risk of impaired healing and osteomyelitis even without treatment with bisphosphonates. We stratified the ROR by sex and age for impaired healing and osteomyelitis associated with alendronate which has the largest market share of bisphosphonates and has the long term data. Effect modification was tested by the Breslow-Day test for homogeneity between odds ratios after stratified analyses. Confounding was assessed by Mantel-Haenszel (M-H) common odds ratios (Table 3). The ROR for impaired healing and osteomyelitis associated with alendronate were unevenly distributed among patients 60 year-old or above and patients less than 60 year-old. The signal of impaired healing and osteomyelitis associated with alendronate after $\mathrm{M}-\mathrm{H}$ age adjustification was significant (Age Adjusted ROR for impaired healing:2.63; for osteomyelitis:2.38). Similarly, the signal of impaired healing and osteomyelitis associated with alendronate after $\mathrm{M}-\mathrm{H}$ sex adjustification was also significant (Sex Adjusted ROR for impaired healing:2.49; for osteomyelitis:2.42). Furthermore, we stratified the ROR of impaired healing and osteomyelitis associated with alendronate over time from 2004 to 2012. Although the total adverse events reported for alendronate has been increasing significantly since 2007(Figure 4A), the annual ROR of impaired healing and osteomyelitis are declining since 2009 (Figure 4B).

\section{Discussion}

For decades bisphosphonate has been the leading class of pharmacotherapy to prevent and treat osteoclast mediated bone loss in osteoporosis, Paget disease, multiple myeloma, and bone complications of metastatic cancers [17]. Accumulating evidence 
Citation: Feng X, Cai A, Hincapie A, Dong K, Chaing W, et al, et al. (2015) Assessing Risks of Impaired Healing and Osteomyelitis Associated with Bisphosphonates Using FDA Adverse Event Reporting System (FAERS). J Pharmacovigilance 3: 158. doi:10.4172/2329-6887.1000158

Page 4 of 6

\begin{tabular}{|c|c|c|c|}
\hline Agent & ROR (95\% Cl) & P -value & PRR \\
\hline Alendronate & $12.109(11.581-12.662)$ & $<0.0005$ & 12.00 \\
\hline Etidronate & $10.173(5.456-18.967)$ & $<0.0005$ & 10.9 \\
\hline Ibandronate & $4.810(4.207-5.514)$ & $<0.0005$ & 4.80 \\
\hline Pamidronate & $7.057(6.608-7.536)$ & $<0.0005$ & 7.02 \\
\hline Risedronate & $8.092(7.095-9.231)$ & $<0.0005$ & 8.04 \\
\hline Zolendronic Acid & $8.849(8.479-9.235)$ & $<0.0005$ & 8.79 \\
\hline
\end{tabular}

$\mathrm{ROR}=$ Reporting odds ratio, $\mathrm{PRR}=$ Proportional reporting ratio

Table 2: Signals of Impaired Healing Associated with Bisphosphonates (First 2004-Quarter to Second 2012-Quarter).

Impaired healing reports for anti-osteoporosis treatments (2004-2012 Q2)

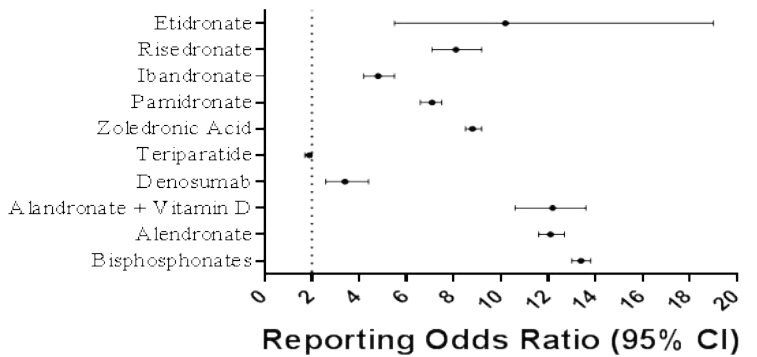

Figure 2: Differential risk of impaired healing associated with bisphosphonates and non-bisphosphonates. The data were obtained from the FAERS from the first quarter of 2004 to second quarter of 2012. Risk of bisphosphonates class was calculated by combining the adverse events of alendronate, risedronate, ibandronate, zoledronic acid, tiludronate and etidronate.

\section{Osteomyelitis reports for anti-osteoporosis} treatments (2004 Q1 2012 Q2)

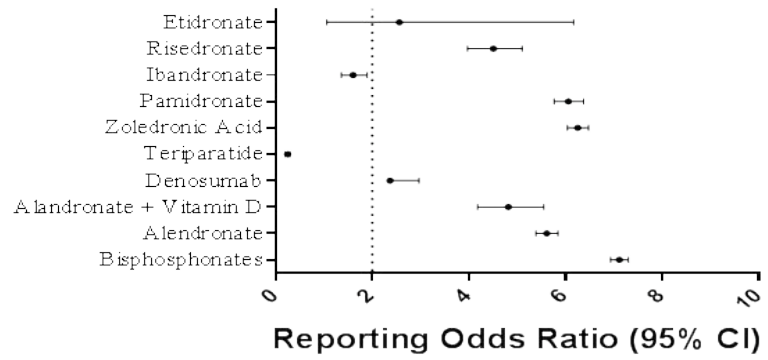

Figure 3: Differential risk of osteomyelitis associated with bisphosphonates and non-bisphosphonates. The data were obtained from the FAERS from the first quarter of 2004 to second quarter of 2012. Risk of bisphosphonates class was calculated by combining the adverse events of alendronate, risedronate, ibandronate, zoledronic acid, tiludronate and etidronate. Bisphosphonates, except ibandronates, demonstrated higher risk of osteomyelitis compared to non-bisphosphonates.

indicates that substantial and significant risks of bone fractures and bone necrosis, particularly osteonecrosis of the jaws (ONJ) are associated with bisphosphonates treatment [18-21]. Assessing the FDA Adverse Event Reporting System (FAERS) public database from the first quarter of 2004 to the second quarter of 2012, for the first time we demonstrated here that there was a significant risk of impaired healing and osteomylitis associated with the use of bisphosphonates.

All the FDA approved bisphosphonates, such as alendronate, pamidronate, risedronate, and etidronate, are associated with significant risk of impaired healing. There are no data available for tilutdronate. Bisphosphonates preferably bind and accumulate in skeletal sites with active turnover remodeling, such as the maxillary and mandibular bones. In physiological condition, sequential activation of osteoclasts and osteoblasts will heal the open bone wounds during the dental procedures quickly and without infection. In contrast, for patients treated with bisphosphonates, especially with large intravenous dose in cancer patients, the accumulating bisphosphonates will be released after tooth extraction and sequentially induce apoptosis of osteoclast and inhibit osteoclast mediated bone resorption in the open bone wound. Thus accumulating bisphosphonates increase risk of open bone wound healing and lead to osteomylitis and necrosis of the skeletal sites [22]. All bisphosphonates have different profile of bone mineral binding capacity and inhibitory effects on osteoclast, which might explain their differences in efficacy and profile of adverse events.

Angiogenesis plays an essential role in wound healing and inhibiting angiogenesis impairs wound healing [23]. Numerous in vitro evidences indicate that bisphosphonates, including ibandronate, have potential inhibitory effect on angiogenesis [24]. Ibandronate has less anti-angiogenic effect compared to pamidronate and zolendronate [25]. This is consistent with our results that ibandronate was associated with the least risk of impaired healing ( $\mathrm{PRR}=4.80)$ compared to higher risks associated with alendronate $(\mathrm{PRR}=12.00)$, zoledronic acid $(\mathrm{PRR}=8.79)$, pamidronate $(\mathrm{PRR}=7.02)$, and risedronate $(\mathrm{PRR}=8.04)$. Although the mechanisms of impaired healing associated with bisphosphonates are not well understood, it is well known that delayed open bone wound healing increases risk of osteomylitis. For the first time we demonstrated that there was a substantial and statistically significant risk of osteomylitis associated with pharmacotherapy of bisphosphonates (except ibandronate) based on the assessment of adverse events reported to FAERS. Ibandronate had minimal associated risk of osteomytitis $(\mathrm{PRR}=1.61)$, compared to those of alendronate $(\mathrm{PRR}=5.57)$, zoledronic acid $(\mathrm{PRR}=6.21)$, pamidronate $(\mathrm{PRR}=6.01)$, and risedronate $(\mathrm{PRR}=4.48)$. This result was consistent with the finding that ibandronate was associated with less risk of impaired healing in comparison with other bisphosphonates. Furthermore, our data also indicated that very weak signal of osteomyelitis was associated with denosumab ( $\mathrm{PRR}=2.38)$, and no significant risk of osteomyelitis were detected for teriparatide $(P R R=0.25)$. The mechanism is still unknown.

FAERS provides an important tool for postmarketing surveillance of less common adverse events associated with pharmacotherapy. The major limitation of the FAERS is underreporting. There is an urgent need to develop social media or mobile tools to encourage consumers and health care professionals to file adverse event report to the FAERS. In addition, the information in the FAERS can be incomprehensible and unsearchable for most consumers and health care providers, since it does not filter, correct, or make any analysis of the quality or potential bias of inputted data. Because the case reporting for the FAERS is spontaneous, there are potential limitations of reporting biases, incomplete reporting, under reporting, and Weber-effect. Furthermore, significant portion of the adverse events were reported with incomplete information, such as drug dose, which make the stratified analysis very difficult [3].

Bisphosphonates are mainly prescribed for female patients with postmenopausal osteoporosis. In addition, chronic wounds and associated infection are more frequent in the elderly. Thus, sex and age are two potential confounding factors that might be associated with bisphosphonates, impaired healing and osteomyelitis. In this study the risk analysis was stratified by age (less than 60 year-old vs. 60 year-old and above) and sex for impaired healing and osteomyelitis associated with alendronate which has the largest market share of 
Citation: Feng X, Cai A, Hincapie A, Dong K, Chaing W, et al, et al. (2015) Assessing Risks of Impaired Healing and Osteomyelitis Associated with Bisphosphonates Using FDA Adverse Event Reporting System (FAERS). J Pharmacovigilance 3: 158. doi:10.4172/2329-6887.1000158

\begin{tabular}{|c|c|c|c|c|}
\hline \multirow{2}{*}{ Factor } & \multicolumn{2}{|c|}{ Impaired healing } & \multicolumn{2}{c|}{ Osteomyelitis } \\
\cline { 2 - 5 } & ROR & $\mathbf{9 5 \%} \mathbf{~ C ~}$ & ROR & $\mathbf{9 5 \%}$ Cl \\
\hline \multicolumn{5}{|c|}{ Age } \\
\hline$\geq 60$ & 4.16 & $3.88-4.47$ & 4.18 & $3.89-4.48$ \\
\hline M-H Age-adjusted analysis & 2.04 & $1.926-2.18$ & 1.86 & $1.76-1.97$ \\
\hline \multicolumn{5}{|c|}{ Sex } \\
\hline Female & 2.63 & $2.51-2.75$ & 2.38 & $2.28-2.49$ \\
\hline Male & 2.50 & $2.37-2.62$ & 1.76 & $1.67-1.85$ \\
\hline M-H Sex-adjusted analysis & 2.43 & $2.11-2.80$ & 8.93 & $8.25-9.66$ \\
\hline & 2.49 & $2.37-2.61$ & 2.42 & $2.32-2.53$ \\
\hline
\end{tabular}

ROR=Reporting odds ratio, *Mantel-Haenszel common odds ratio.

Table 3: Risk of Impaired Healing and Osteomyelitis Associated with Alendronate Stratified by Age and Sex.

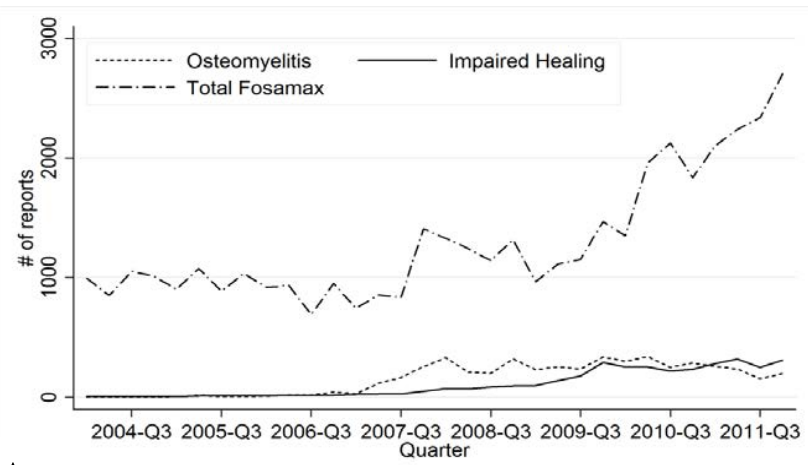

A

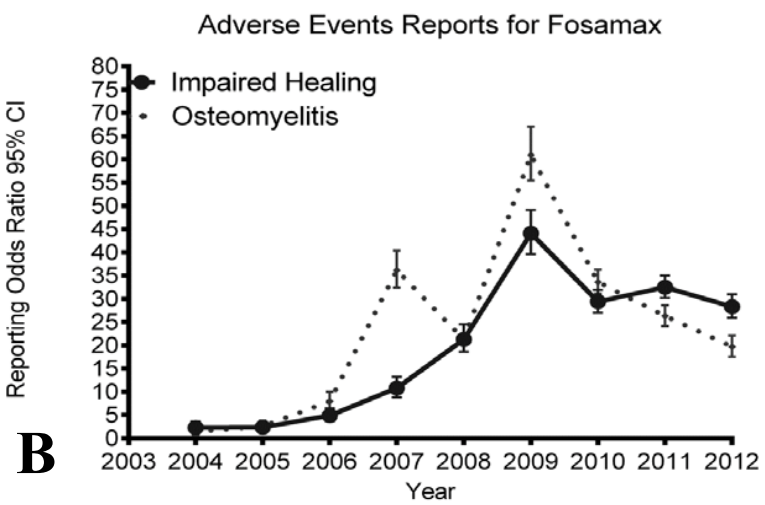

Figure 4: Stratified Analysis for Risk of Impairing Healing and Osteomyelitis Associated with Alendronate over Time. The data were obtained from the FAERS from the first quarter of 2004 to second quarter of 2012. 4A) Total cases of all adverse events, adverse events of impaired healing and adverse events of osteomyelitis associated with alendronate. 4B) Stratified reporting odds ratio (ROR) for impaired healing and osteomyelitis from 2004 to 2012.

bisophosphonates and long term post market data. The risk signals of impaired healing and osteomyelitis associated with alendronate after age and sex stratification were still significant. Furthermore, there is concern of reporting biases for impaired healing and osteomyelitis associated with bisophosphonates, since the FDA recently issued drug safety warning of osteonecrosis of jaw (ONJ) and bisphosphonateassociated atypical femoral fractures. Although the total adverse events associated with bisphosphonates are increasing substantially from 2004 to 2012 in FAERS, the ROR for impaired healing and osteomyelitis starts to decline since 2009. One of the possible explanations is that clinicians are more cautious to use bisphosphonates for patients with risk of chronic wound, especially for long term use.

\section{Conclusion}

Since the case reporting for the FAERS is spontaneous, there are potential limitations of reporting biases, incomplete reporting, under reporting, and Webb-effect. Although randomized, controlled clinical trials remain the gold standard to assess pre-market and post-market drug safety; pharmacovigilance systems like the FAERS plays a critical role to raise the clinical and public awareness of the potential risks of impaired healing and osteomylitis associated with biosphosphonates, especially for long term use and high dose treatment with bisphosphonates. This study also provided goal for future large scale pharmacoepidemiologic studies and provided directions for clinical trials with better control of confounding factors.

\section{References}

1. Banerjee AK, Ingate $S$ (2012) Web-based patient-reported outcomes in drug safety and risk management: challenges and opportunities?. Drug Saf 35: $437-$ 446.

2. Borg JJ, Aislaitner G, Pirozynski M, Mifsud S (2011) Strengthening and rationalizing pharmacovigilance in the EU: where is Europe heading to? A review of the new EU legislation on pharmacovigilance. Drug Saf 34: 187-197.

3. Hoffman KB, Kraus C, Dimbil M, Golomb BA (2012) A survey of the FDA's AERS database regarding muscle and tendon adverse events linked to the statin drug class. PLoS One 7: e42866.

4. Piccinni C, Motola D, Marchesini G, Poluzzi E (2011) Assessing the association of pioglitazone use and bladder cancer through drug adverse event reporting. Diabetes Care 34: 1369-1371.

5. Van Beusekom HM, Saia F, Zindler JD, Lemos PA, Swager-Ten Hoor SL, et al. (2007) Drug-eluting stents show delayed healing: paclitaxel more pronounced than sirolimus. Eur Heart J 28: 974-979.

6. Colombres GA, Gramajo AL, Arrambide MP, Juarez SM, Arevalo JF, et al. (2011) Delayed corneal epithelial healing after intravitreal bevacizumab: a clinical and experimental study. J Ophthalmic Vis Res 6: 18-25.

7. Russell RG, Watts NB, Ebetino FH, Rogers MJ (2008) Mechanisms of action of bisphosphonates: similarities and differences and their potential influence on clinical efficacy. Osteoporos Int 19: 733-759.

8. Papapetrou PD (2009) Bisphosphonate-associated adverse events. Hormones (Athens) 8: 96-110.

9. Kennel KA, Drake MT (2009) Adverse effects of bisphosphonates: implications for osteoporosis management. Mayo Clin Proc 84: 632-638.

10. Stein EM, Ortiz D, Jin Z, McMahon DJ, Shane E (2011) Prevention of fractures after solid organ transplantation: a meta-analysis. J Clin Endocrinol Metab 96 3457-3465.

11. Edwards BJ, Bunta AD, Lane J, Odvina C, Rao DS, et al. (2013) Bisphosphonates and Nonhealing Femoral Fractures: Analysis of the FDA Adverse Event Reporting System (FAERS) and International Safety Efforts: A Systematic Review from the Research on Adverse Drug Events And Reports (RADAR) Project. J Bone Joint Surg Am 95: 297-307.

12. Almenoff JS, Pattishall EN, Gibbs TG, DuMouchel W, Evans SJ, et al. (2007) Novel statistical tools for monitoring the safety of marketed drugs. Clin Pharmacol Ther 82: 157-166

13. Evans SJ, Waller PC, Davis S (2001) Use of proportional reporting ratios (PRRs) for signal generation from spontaneous adverse drug reaction reports. Pharmacoepidemiol Drug Saf 10: 483-486.

14. van Puijenbroek EP, Bate A, Leufkens HG, Lindquist $M$, Orre $R$, et al. (2002) A comparison of measures of disproportionality for signal detection in spontaneous reporting systems for adverse drug reactions. Pharmacoepidemiol Drug Saf 11: 3-10.

15. Bate A, Lindquist M, Edwards IR, Olsson S, Orre R, et al. (1998) A Bayesian neural network method for adverse drug reaction signal generation. Eur J Clin Pharmacol 54: 315-321. 
Citation: Feng X, Cai A, Hincapie A, Dong K, Chaing W, et al, et al. (2015) Assessing Risks of Impaired Healing and Osteomyelitis Associated with Bisphosphonates Using FDA Adverse Event Reporting System (FAERS). J Pharmacovigilance 3: 158. doi:10.4172/2329-6887.1000158

Page 6 of 6

16. Szarfman A, Machado SG, O'Neill RT (2002) Use of screening algorithms and computer systems to efficiently signal higher-than-expected combinations of drugs and events in the US FDA's spontaneous reports database. Drug Saf 25: 381-392.

17. Michaelson MD, Smith MR (2005) Bisphosphonates for treatment and prevention of bone metastases. J Clin Oncol 23: 8219-8224.

18. Boonyapakorn T, Schirmer I, Reichart PA, Sturm I, Massenkeil G (2008) Bisphosphonate-induced osteonecrosis of the jaws: prospective study of 80 patients with multiple myeloma and other malignancies. Oral Oncol 44: 857-869.

19. Sedghizadeh PP, Stanley K, Caligiuri M, Hofkes S, Lowry B, et al. (2009) Oral bisphosphonate use and the prevalence of osteonecrosis of the jaw: an institutional inquiry. J Am Dent Assoc 140: 61-66.

20. Khosla S, Burr D, Cauley J, Dempster DW, Ebeling PR, et al. (2007) American Society for Bone and Mineral Research: Bisphosphonate-associated osteonecrosis of the jaw: report of a task force of the American society for bone and mineral research. J Bone Miner Res 22: 1479-1491.
21. Ruggiero SL, Dodson TB, Assael LA, Landesberg R, Marx RE, et al. (2009) American Association of Oral and Maxillofacial Surgeons Position Paper on Bisphosphonate-Related Osteonecrosis of the Jaw-2009 Update. Australian endodontic journal 35: 119-130.

22. Silverman SL, Landesberg $R$ (2009) Osteonecrosis of the jaw and the role of bisphosphonates: a critical review. Am J Med 122: S33-45.

23. Tonnesen MG, Feng X, Clark RA (2000) Angiogenesis in wound healing. J Investig Dermatol Symp Proc 5: 40-46.

24. Sharma D, Ivanovski S, Slevin M, Hamlet S, Pop T, et al. (2013) Bisphosphonate-related osteonecrosis of jaw (BRONJ): diagnostic criteria and possible pathogenic mechanisms of an unexpected anti-angiogenic side effect. Vascular Cell 5: 1-8.

25. Ziebart T, Pabst A, Klein MO, Kämmerer P, Gauss L, et al. (2011) Bisphosphonates: restrictions for vasculogenesis and angiogenesis: inhibition of cell function of endothelial progenitor cells and mature endothelial cells in vitro. Clin Oral Investig 15: 105-111. 